\title{
No Influence of Type 2 Diabetes on Chronic Inflammation and Oxidative Stress in Obese Patients
}

\author{
Adriana Florinela CĂTOI ${ }^{1}$, Alina Elena PÂRVU²*, Adriana MUREŞAN ${ }^{1}$, \\ Cristina BIDIAN ${ }^{1}$, Cornel CĂTOI ${ }^{3}$, Ioana Delia POP $^{4}$ \\ 'Department of Physiology, Faculty of Medicine, "Iuliu Haţieganu" University of Medicine and Pharmacy "Iuliu Hatieganu" Cluj-Napoca, RO- \\ 400012, Cluj-Napoca, Romania \\ ${ }^{2}$ Department Pathophysiology, Faculty of Medicine, "Iuliu Hatieganu" University of Medicine and Pharmacy Cluj-Napoca, RO- \\ 400012,Cluj-Napoca,Romania;parvualinaelena@yahoo.com \\ ( ${ }^{*}$ corresponding author; the first two authors have equal contribution to the study) \\ ${ }^{3}$ Department of Pathology, Veterinary Medicine Faculty, University of Agricultural Sciences and Veterinary Medicine Cluj-Napoca, \\ RO-400372, Cluj-Napoca, Romania \\ ${ }^{4}$ Department of Exact Sciences, Horticulture Faculty, University of Agricultural Sciences and Veterinary Medicine Cluj-Napoca, RO- \\ 400372 Cluj-Napoca, Romania
}

\begin{abstract}
Obesity per se carries the features of chronic inflammation and oxidative stress that interrelate in a complex network and exert an important role in the onset of several complications such as type 2 diabetes, atherosclerosis and cardiovascular events. On the other hand, it seems that hyperglycemia per se as well as insulin resistance (independent of hyperglycemia), both induce increased oxidative stress. The aim of our study was to analyze proinflammatory and oxidative stress markers in obese patients with and without type 2 diabetes and to verify the hypothesis that type 2 diabetes associated with obesity would promote a higher chronic inflammation and oxidative stress state as compared to obesity alone. We found no differences between the two groups of patients regarding chronic inflammation and oxidative stress markers. Therefore we may conclude that there is no influence of type 2 diabetes on chronic inflammation and oxidative stress in obese patients.
\end{abstract}

Keywords: chronic inflammation, obesity, oxidative stress, type 2 diabetes mellitus

\section{Introduction}

Obesity is increasing worldwide at an alarming rate (Low et al., 2009). In Romania, the available epidemiological data revealed a $20-25 \%$ prevalence of obesity (Cinteză et al., 2007). Obesity is associated with several diseases such as type 2 diabetes (T2DM), hypertension, dyslipidemia, coronary heart disease, stroke, osteoarthritis, sleep apnea and different malignances which increase cardiovascular risk, mortality and consecutively reduces life expectancy (Buchwald et al., 2004).

Adipose tissue is known to be an endocrine organ producing adipokines which include hormones such as leptin or adiponectin and inflammatory cytokines such as tumor necrosis factor alpha (TNF- $\alpha$ ) or interleukin 6 (IL-6) among others (Wang and Nakayama, 2010; Maury and Brichard, 2010). They are dysregulated in obesity and contribute to the development of obesitylinked metabolic disorders and cardiovascular diseases
(Maury and Brichard, 2010; Bullo et al., 2006; Wozniak et al., 2009). A large body of evidence showed that lowgrade chronic inflammation found in obesity increases the expression and activity of prooxidant enzymes including myeloperoxidase and NADPH oxidases and promote the production of reactive free radicals (Park $e t$ al., 2007). On the other hand, antioxidant defense markers are lower in obesity according to the amount of body fat and central obesity (Iyer $e t$ al., 2010). Leptin is a hormone secreted by adipocytes in direct proportion to the mass of adipose tissue, promoting oxidative stress and vascular inflammation in obesity, therefore favoring the development of atherosclerosis (Fernández-Sánchez and Madrigal-Santillán, 2011).

Noteworthy, that obesity per se induces oxidative stress due to an increase production of free radicals by the excess adipose tissue (Furukawa et al., 2004). Hence, chronic inflammation and oxidative stress that occur in obesity interrelate and have an important role in triggering and exacerbating its associated pathologic conditions such as insulin resistance, hyperglycemia and 
metabolic dysfunction (Iyer et al., 2010; FernándezSánchez and Madrigal-Santillán, 2011). On the other hand, several studies have hypothesized that hyperglycemia per se as well as insulin resistance (independent of hyperglycemia) both induce increased oxidative stress through complex mechanisms that exert an important role in the onset of diabetic complications such as atherosclerosis and cardiovascular events. (Grattagliano et al., 2008; Stephens et al., 2009; Avogaro and de Kreutzenberg, 2005). Taken together, assuming that obesity, insulin resistance and hyperglycemia together promote a even higher state of chronic inflammation and oxidative stress versus obesity alone, it is difficult to conclude whether there is first an increase of these parameters and later the onset of T2DM or whether T2DM is responsible for the even more elevated levels of pro-inflammatory and oxidative stress markers in obesity.

\section{Materials and methods}

\section{Research design, Setting and Institutional Review}

The present research was carried out as a prospectively controlled clinical study and the data collection period was between January 2009 and February 2011. It was conducted at the Physiology Department of "Iuliu Haţieganu" University of Medicine and Pharmacy Cluj-Napoca, Romania. The study protocol was approved by the Medical Ethics Committee of "Iuliu Hațieganu" University of Medicine and Pharmacy Cluj-Napoca, Romania in accordance with the ethical principles of the World Medical Association Declaration of Helsinki (Bulletin of the World Health Organization, 2001). All the patients agreed to be included in this study and gave their written informed consent

\section{Study Subjects}

Thirty-four obese patients ( 24 female and 10 male) aged between 20 and 59 years and a mean BMI (body mass index) of $48.59 \pm 7.86 \mathrm{~kg} / \mathrm{m}^{2}$ were recruited for this study. All the patients were selected from the Second Surgical Clinic form Cluj-Napoca and were eligible for bariatric surgery. They were divided into one subgroup consisting in 17 patients formed out of patients with T2DM (10 female and 7 male) and another subgroup of 17 obese patients without T2DM (14 female and 3 male). Inclusion criteria were BMI $\geq 40 \mathrm{~kg} / \mathrm{m}^{2}$ or $>35$ $\mathrm{kg} / \mathrm{m}^{2}$ with associated co-morbidities and approval to participate to the present study. Exclusion criteria were: physical disorders, smoking, alcohol or drug addiction, severe endocrine diseases other than diabetes, infectious and chronic inflammatory diseases, no clinical evidence of severe cardiac, hepatic, or renal failure, neoplasia or chronic systemic diseases. None of the patients were on antidiabetic medication.

Twenty normal weight healthy subjects (10 female and 10 male) aged between 25 and 50 years and a mean BMI of $22.09 \pm 3.37 \mathrm{~kg} / \mathrm{m}^{2}$ formed the control group. They were clinically and biochemically free from any disease.
The diagnostic of T2DM was based on fasting glucose value $\geq 126 \mathrm{mg} / \mathrm{dl}$ or blood glucose $\geq 200 \mathrm{mg} / \mathrm{dl} 2 \mathrm{~h}$ after an OGTT.from the third) were mixed and the final extract of flower and leaf respectively were 1:1 (w:v) respectively (Pârvu et al., 2010a).

\section{Variables, Blood collection, and Analytical Methods}

BMI was calculated by dividing the weight in kilograms by the square of the height in meters.

Blood samples were obtained after overnight fast, collected into tubes and serum was separated after centrifugation at $1500 \mathrm{~g}$ for 10 minutes. Serum samples were analyzed immediately or stored at $-80{ }^{\circ} \mathrm{C}$. They were run at the Clinical Laboratory of the Second Surgical Clinic, Cluj-Napoca and at the Oxidative Stress Laboratory from the Physiology Department of "Iuliu Haţieganu" University of Medicine and Pharmacy ClujNapoca, Romania.

Fasting glucose, total cholesterol and triglycerides were determined using the standard enzymatic colorimetric methods (Clini-Lab Diagnosticum Hungary). Serum HDL-cholesterol (HDL-C) was determined using the precipitation method (Clini-Lab Diagnosticum Hungary). The device used was Cobas Myra Plus Roche Switzerland. Serum LDL-cholesterol (LDL-C) concentration was calculated by the Friedewald formula (Friedewald et al., 1972). Insulin was essayed from serum by electrochemiluminescence using Roche kits (Basel, Switzerland) and the Roche Elecsys 1010 immunoassay analyzer. Insulin resistance was evaluated using the homeostatic model assessment for insulin resistance (HOMA-IR) and was calculated using the following formula: HOMA IR= (fasting glucose $\mathrm{mg} / \mathrm{dl} \times$ fasting insulin $\mu \mathrm{U} / \mathrm{ml}) / 22.5 \times 18$. A normal value was considered to be $\leq 2.5$ (Matthews et al., 1985). Leptin levels were measured from serum by enzyme immunoassay (ELISA) using a EIA395 DRG Diagnostics kit (Marburg, Germany) and Organon 230 S Oss Olanda reader. Concentration of tumor necrosis factor alpha (TNF- $\alpha$ ) was determined by ELISA method using the EIA-3134 DRG kit and Organon 230 S Oss Olanda reader.

Determination of the serum products of lipoperoxidation (LPO) was based on the evaluation of the colored product that results after the reaction between malondialdehyde (MDA) with thiobarbituric acid. Results were expressed as $\mathrm{nmol} / \mathrm{ml}$ serum (Esterbauer and Chjeeseman, 1994).

The assay of the hydrogen donor ability (HAD) of the serum was performed using the Janazewska biochemical method (Janazsewska and Bartosz, 2002). This method is based on the reduction of the free stable 1,1-diphenyl-picrylhydrazyl (DPPH) by several non enzymatic antioxidant compounds of the serum like glutathione, tocoferol, ascorbic acid.

This reduction can be observed through the change of color from violet to pale yellow, monitored by the change of the absorbance at $520 \mathrm{~nm}$. Hydrogen donor ability was measured in \% inhibition compared to control samples according to the following formula: 
16

Inhibition $\%=[($ control sample absorbance- serum sample absorbance)/control sample absorbance] X 100.

\section{Statistical analysis}

The univariate normality of the data from the obese patients and from control subjects, respectively, was analyzed using the Shapiro-Wilk test. Data was expressed as median and interquartile range for non-parametric variables and as mean \pm standard deviation for parametric variables. The comparison between two independent groups was performed using the Student's $t$ test associated with the Levene test for determining the homogeneity of the variance for the normally distributed data. The Mann-Whitney $U$-test was applied for comparison between two independent groups for non parametric variables. When analyzing the subgroups the multiple comparison correction of Bonferroni was used in order to keep the error rate $(\alpha)$ to the specified level of 0.05. Spearman's test was used to evaluate the relationships between variables. Levels of statistical significance were set at $P<0.05$. The statistical analysis was performed with the SPSS v.19 software.

\section{Results}

\section{Serum total oxidant status determination}

The characteristics of the groups were presented in Tab. 1. No statistically significant differences were observed between controls and obese patients concerning total cholesterol and LDL-cholesterol, and HDA.

Tab. 1. Anthropometric and laboratory characteristics of the groups

\begin{tabular}{|c|c|c|c|}
\hline Parameters & Control $(n=20)$ & Obese $(n=34)$ & $\mathrm{p}$ \\
\hline Age & $32.25 \pm 8.97$ & $36.48 \pm 12.42$ & NS \\
\hline $\mathrm{BMI}\left(\mathrm{kg} / \mathrm{m}^{2}\right)$ & $22.00 \pm 3.37$ & $48.25(41.88-53.60)$ & $\mathrm{p}<0.001$ \\
\hline Fasting glucose $(\mathrm{mg} / \mathrm{dl})$ & $78.63 \pm 16.05$ & $114.50(99.00-135.25)$ & $\mathrm{p}<0.001$ \\
\hline Total cholesterol $(\mathrm{mg} / \mathrm{dl})$ & $183 \pm 33.73$ & $213.06 \pm 46.42$ & NS \\
\hline $\mathrm{HDL}-\mathrm{C}(\mathrm{mg} / \mathrm{dl})$ & $56.50 \pm 7.76$ & $43.69 \pm 11.35$ & $\mathrm{p}<0.05$ \\
\hline LDL-C (mg/dl) & $106.90 \pm 27.07$ & $136.43 \pm 46.66$ & NS \\
\hline Triglycerides $(\mathrm{mg} / \mathrm{dl})$ & $98.00 \pm 28.96$ & $149(113.00-195.00)$ & $\mathrm{p}<0.05$ \\
\hline LDL-C/HDL-C & $2.04(1.83-2.20)$ & $3.13(2.52-3.96)$ & $\mathrm{p}<0.05$ \\
\hline Insulin $(\mu \mathrm{U} / \mathrm{mL})$ & $9.07 \pm 3.19$ & $29.21 \pm 12.83$ & $\mathrm{p}<0.001$ \\
\hline HOMA-IR & $1.77 \pm 0.82$ & $8.72 \pm 4.13$ & $\mathrm{p}<0.001$ \\
\hline Leptin $(\mathrm{ng} / \mathrm{ml})$ & $12.97 \pm 4.24$ & $55.23(44.36-79.49)$ & $\mathrm{p}<0.001$ \\
\hline $\mathrm{TNF}-\alpha(\mathrm{pg} / \mathrm{ml})$ & $9.10 \pm 4.63$ & $16.01(12.60-28.08)$ & $\mathrm{p}<0.001$ \\
\hline $\mathrm{LPO}(\mathrm{nmol} / \mathrm{ml})$ & $1.70(1.43-1.92)$ & $2.61(2.09-3.45)$ & $\mathrm{p}<0.001$ \\
\hline $\mathrm{HDA} \%$ & $46.65 \pm 5.35$ & $40.08 \pm 13.67$ & NS \\
\hline
\end{tabular}

Tab. 2. Anthropometric and laboratory characteristics of the subgroups

\begin{tabular}{|c|c|c|c|}
\hline Parameters & Control $(n=20)$ & Obese with T2DM $(\mathrm{n}=17)$ & Obese without T2DM $(n=17)$ \\
\hline Age & $32.25 \pm 8.97$ & $44.41 \pm 9.97$ & $24(21.25-36.50) * c$ \\
\hline $\operatorname{BMI}\left(\mathrm{kg} / \mathrm{m}^{2}\right)$ & $22.00 \pm 3.37$ & $51.76 \pm 6.21^{*_{a}}$ & $42.24(39.88-50.20)^{* \mathrm{~b}}$ \\
\hline Fasting glucose (mg/dl) & $78.63 \pm 16.05$ & $110.46 \pm 37.16^{* a}$ & $98.47 \pm 9.00^{* \mathrm{~b}, \mathrm{c}}$ \\
\hline Total cholesterol $(\mathrm{mg} / \mathrm{dl})$ & $183 \pm 33.73$ & $216.88 \pm 55.31$ & $209.47 \pm 37.61$ \\
\hline HDL-C $(\mathrm{mg} / \mathrm{dl})$ & $56.50 \pm 7.76$ & $44.09 \pm 11.75$ & $43.34 \pm 11.36$ \\
\hline LDL-C (mg/dl) & $106.90 \pm 27.07$ & $138.33 \pm 56.28$ & $134.78 \pm 38.19$ \\
\hline Triglycerides (mg/dl) & $98.00 \pm 28.96$ & $183.00 \pm 80.98$ & $137(108-166)$ \\
\hline LDL-C/HDL-C & $2.04(1.83-2.20)$ & $2.86(2.29-3.92)$ & $3.26 \pm 1^{* b}$ \\
\hline Insulin $(\mu \mathrm{U} / \mathrm{mL})$ & $9.07 \pm 3.19$ & $32.12 \pm 9.14^{* a}$ & $21.53(14.83-36.92)^{* b}$ \\
\hline HOMA-IR & $1.77 \pm 0.82$ & $11.03 \pm 3.11^{*_{a}}$ & $6.42 \pm 3.76^{* \mathrm{~b}, \mathrm{c}}$ \\
\hline Leptin (ng/ml) & $12.97 \pm 4.24$ & $55.70(46.35-80.79)^{* a}$ & $54.76(40.11-79.86)^{* b}$ \\
\hline TNF- $\alpha(\mathrm{pg} / \mathrm{ml})$ & $9.10 \pm 4.63$ & $16.48(14.59-32.37)$ & $15.54(11.04-21.77)$ \\
\hline $\mathrm{LPO}(\mathrm{nmol} / \mathrm{ml})$ & $1.70(1.43-1.92)$ & $2.67(2.36-3.88){ }^{* a}$ & $2.57 \pm 0.86^{* \mathrm{~b}}$ \\
\hline HAD\% & $46.65 \pm 5.35$ & $36.31 \pm 12.61$ & $43.86 \pm 14.12$ \\
\hline
\end{tabular}

The results are expressed as mean $\pm s . d$ for parameters with Gaussian distribution and median (25-75 percentiles) for non Gaussian distribution; $\mathrm{p}$-values were calculated by the Student $\mathrm{t}$ - test for normally distributed variables or the nonparametric Mann-Whitney U-testBMI: body mass index, HDL-C: high-density lipoproteincholesterol, LDL-C: low-density lipoprotein-cholesterol, HOMA-IR: homeostasis model assessment-insulin resistance, TNF- $\alpha$ : tumor necrosis factor $\alpha$, LPO: lipoperoxides, HAD: hydrogen donor ability

$\mathrm{p}<0.0083$ (Bonferroni correction: alfa $=0.05 / 3=0.0016$, differences between groups)

${ }^{\mathrm{a}}$ Differences between the controls and obese with T2DM

${ }^{b}$ Differences between controls and obese without T2DM

${ }^{\mathrm{c}}$ Differences between obese with and without T2DM 
The results are expressed as mean \pm s.d for parameters with Gaussian distribution and median (25-75 percentiles) for non Gaussian distribution; p-values were calculated by the Student $\mathrm{t}$ - test (normally distributed variables; total cholesterol, HDL cholesterol, LDL cholesterol, insulin, HOMA-IR, HDA) or the nonparametric Mann-Whitney U-test (not normally The results are expressed as mean \pm s.d for parameters with Gaussian distribution and median (25-75 percentiles) for non Gaussian distribution; p-values were calculated by the Student $\mathrm{t}$ - test (normally distributed variables; total cholesterol, HDL cholesterol, LDL cholesterol, insulin, HOMA-IR, HDA) or the nonparametric Mann-Whitney U-test (not normally distributed variables; BMI, fasting glucose, triglycerides, leptin, TNF- $\alpha$, LPO). BMI: body mass index, HDL-C: high-density lipoprotein-cholesterol, LDL-C: lowdensity lipoprotein-cholesterol, HOMA-IR: homeostasis model assessment-insulin resistance, TNF- $\alpha$ : tumor necrosis factor $\alpha$, LPO: lipoperoxides, HAD: hydrogen donor ability.

A statistically significant positive correlation between BMI, HOMA-IR $(\mathrm{r}=0.477, \mathrm{p}<0.05)$ and insulin $(\mathrm{r}=0.405, \mathrm{p}<0.05)$ respectively was noticed in the group of obese patients. Also, we observed a significant positive correlation between fasting glucose and LPO $(r=0.372$, $\mathrm{p}<0.05)$ as well as a statistically significant negative correlation between fasting glucose and HAD $(r=-0.399$, $\mathrm{p}<0.05)$ in the obese group.

Tab. 2 shows the values of the controls as compared with the two subgroups of obese patients with and without type 2 diabetes. Obese patients with type T2DM had a higher BMI $(p<0.0016)$, fasting glucose $(\mathrm{p}<0.0016)$ and HOMA-IR $(\mathrm{p}<0.0016)$ and as compared to the group of obese patients without T2DM. There were no differences between the two groups of obese with and without T2DM regarding circulating levels of leptin, TNF- $\alpha$, LPO and HAD. A difference was noticed between the two groups regarding insulin, although it was not statistically significant.

No significant correlations were noticed in the subgroup of obese patients without T2DM. Only one significant correlation was observed in the T2DM obese group, i.e between leptin and HDL-C $(r=-0.67, \mathrm{p}<0.05)$.

\section{Discussions}

The present study aimed to investigate the influence of T2DM on markers of chronic inflammation and oxidative stress in obese patients, in order to verify the hypothesis that T2DM associated with obesity would significantly increase the aforementioned pathological state. In the large group of patients we confirmed that obese both with and without T2DM carry the proinflammatory and a pro-oxidant phenotype, although HDA was not significantly decreased as compared to controls, showing that there still exists an important potential source of antioxidant defence within the body.

Oxidative stress occurs as a consequence of unbalanced ratio between of free radicals production and enzymatic/ non enzymatic anti-oxidative defense system.
The main reactive oxigen specie is superoxide anion $\left(\mathrm{O}_{2}{ }^{\circ}\right)$ produced by NADPH oxidase, a highly reactive and cytotoxic agent (Hopps et al., 2010). When overproduced it could react with macromolecules inducing lipid peroxidation, protein and nucleic acid modifications as well as DNA damage (Roberts and Sindhu, 2009). Oxidative stress is promoted and perpetuated by chronic inflammation, thereby inducing a vicious cycle (Iyer et al., 2010). Obesity per se is asociated with the features of chronic inflammation and increased oxidative stress and a large body of evidence has emphasized the involvement of these mechanisms in the developement of type 2 diabets mellitus, atherosclerosis and cardiovascular events (Iyer et al., 2010; FernándezSánchez and Madrigal-Santillán, 2011; Furukawa et al., 2004). Noteworthy, that nutrient excess in obese patients might also contribute to increased oxidative stress (Sledzinski et al., 2009).

In order to evaluate the influence of T2DM we divided the obese patients in two subgroups with and without the aforementioned disease and we assumed that obese with T2DM would have an increased level of chronic inflammation and oxidative stress markers as compared to the obese without T2DM. Although significant differences regarding HOMA-IR values were revealed, we observed no variations regarding TNF- $\alpha$, LPO and HDA. However, we have to mention that our patients followed no medication for diabetes, the blood glucose levels were not extremely increased, some of them were newly diagnosed and others had a short history of diabetes and no complications.

Consistent with our data, Kopp et al. (2003) after investigating the relationship between circulating levels of pro-inflammatory proteins in morbidly obese patients selected for bariatric surgery, according to glucose tolerance, revealed that concentrations of TNF- $\alpha$ showed no differences between patients ranging from normal glucose tolerance to T2DM (Kopp et al., 2003). In line with our results, Catalan et al. (2007) analyzed the impact of T2DM on proinflammatory cytokines in obesity and pointed out that these parameters relate mainly to obesity rather than T2DM. However, they could not rule out the involvement of inflammation in T2DM development (Catalan et al., 2007). Interestingly, in our subgroups we did not notice any significant correlations between obesity, markers of chronic inflammation, oxidative stress and insulin resistance, so we could not draw a conclusion based on the interrelations between these mechanisms. A recent study analyzed a group of morbidly obese patients with insulin resistance as compared to morbidly obese with T2DM and no antidiabetic treatment and showed that the two groups exhibited the same pro-inflammatory profile. Starting from the assumption that T2DM would be induced by an increased level of chronic inflammation, the authors concluded that other mechanisms might be involved in the onset of T2DM in obesity (Barbarroja et al., 2012).

Insulin resistance and hyperglycemia could induce oxidative stress through processes such as glucose autooxidation, advanced glycation end product formation, or increase of the activity of nitric oxide synthase 
18

(Grattagliano et al., 2008). However, in our study the more elevated levels of insulin and HOMA-IR values found in obese patients with T2DM did not seem to be associated with a significant higher state of oxidative stress as compared with the non diabetic group. Some other authors approached the same topic and demonstrated that severely obese patients (BMI range: $43.3-74 \mathrm{~kg} / \mathrm{m}^{2}$ ) with higher insulin resistance displayed a lower plasma superoxide dismutase (SOD) activity and a greater glutathione peroxidase activity. Still, when looking at TAC (total antioxidant capacity) and TBARS (thiobarbituric acid reactive substances) there were no significant differences between the obese with higher insulin resistance as compared those with lower insulin resistance (Tinahones et al., 2008). On the other hand, Stefanovići et al. (2008) demonstrated that the association between obesity and T2DM exerted a powerful influence on oxidative stress markers, as TBARS were more elevated in these patients in comparison with controls with BMI over $30 \mathrm{~kg} / \mathrm{m}^{2}$ (Stefanović et al., 2008).

Regarding circulating levels of leptin, they are increased in obesity and are postulated to be implicated in increasing low-grade inflammation and in inducing reactive oxigen species production thereby leading to significant oxidative stress (Fernández-Sánchez and Madrigal-Santillán, 2011; Sledzinski et al., 2009). Herein, although the BMI values were slightly different between the two subgroups, we observed no difference regarding circulating leptin levels, which is an accurate marker of the amount of fat mass, suggesting that BMI might not be the ideal tool to evaluate obesity.

Opposite to our findings, Stefanovići et al. (2008) revealed that leptin levels were significantly higher in T2DM obese patients when compared with obese controls, pointing out that the presence of T2DM made the difference (Stefanović et al., 2008).

In this case, the explanation given by the authors concerned the distribution of fat mass, suggesting that intra-abdominal obesity would increase even more leptin production (Stefanović et al., 2008). It could be possible that the difference might occur from the fact that Stefanović et al. (2008) had a lower BMI (29.1 \pm 5.2 $\mathrm{kg} / \mathrm{m}^{2}$ ) as compared to the patients from our study groups (BMI over $35 \mathrm{~kg} / \mathrm{m}^{2}$ ).

\section{Conclusion}

In conclusion, our data has precluded the hypothesis that obese patients with T2DM would carry an increased phenotype of chronic inflammation and oxidative stress as compared with obesity alone, indicating that there seems to be no influence of T2DM on these parameters in obese patients.

\section{Acknowledgements}

This study was performed as part of a financed grant from the University of Medicine and Pharmacy "Iuliu Hatieganu", Cluj-Napoca (Romania), no. 27020/39/15.11.2011.

\section{References}

Avogaro A, de Kreutzenberg SV (2005). Mechanisms of endothelial dysfunction in obesity. Clinica Chimica Act 360:9-26.

Barbarroja N, Lopez-Pedrera C, Garrido-Sanchez L, Mayas MD, Oliva Olivera W, Bernal-Lopez MR, El Bekay R, Tinahones FJ (2012). Progression from High Insulin Resistance to Type 2 Diabetes Does Not Entail Additional Visceral Adipose Tissue Inflammation. PLoS One 7(10):e48155.

Buchwald H, Avidor Y, Braunwald E, Jensen MD, Pories W, Fahrbach K, Schoelles K (2004). Bariatric surgery. JAMA 292(14):1724-1737.

Bullo M, Casas-Agustench P, Amigó-Correig P, Aranceta J, Salas-Salvado J (2006). Inflammation, obesity and comorbidities: the role of diet. Public Health Nutrition 10(10A):1164-1172.

Catalan V, Gomez-Ambrosi J, Ramirez B, Rotellar F, Pastor C, Silva C (2007). Proinflammatory cytokines in obesity: impact of type 2 diabetes mellitus and gastric bypass. Obes Surg 17(11):1464-1474.

Cinteză M, Pană B, Cochino E, Florescu M, Margulescu A, Florian A, Vinereanu D (2007). Prevalence and control of cardiovascular risk factors in Romania cardio-zone national study. Maedica - a Journal of Clinical Medicine 2(4):277282.

Esterbauer H, Chjeeseman K (1994). Determination of aldehydic lipid peroxidation products: malonaldehyde and 4-hydroxynonenal. Methods in Enzymology 186:406-13.

Fernández-Sánchez A, Madrigal-Santillán E (2011). Bautista $\mathrm{M}$ et al. Inflammation, oxidative stress, and obesity. Int $\mathrm{J}$ Mol Sci.12:3117-3132.

Friedewald WT, Levy RI, Fredrickson DS (1972). Estimation of the concentration of low-density lipoprotein cholesterol in plasma, without use of the preparative centrifuge. Clin Chem. 18: 499-500.

Furukawa S, Fujita T, Shimabukuro M, Iwaki M, Yamada Y, Nakajima Y, Nakayama O, Makishima M, Matsuda M (2004). Increased oxidative stress in obesity and its impact on metabolic syndrome. J Clin Invest 114:1752-1761.

Grattagliano I, Palmieri VO, Portincasa P, Moschetta A, Palasciano (2008). Oxidative stress-induced risk factors associated with the metabolic syndrome: a unifying hypothesis. Journal of Nutritional Biochemistry 19:491504.

Hopps E, Noto D, Caimi G, Averna MR (2010). A novel component of the metabolic syndrome: The oxidative stress. Nutrition, Metabolism and Cardiovascular Diseases 20:72-77.

Iyer A, Fairlie DP, Prins JB, Hammock BD, Brown L (2010). Inflammatory lipid mediators in adipocyte function and obesity nature reviews. Endocrinology 6:71-82.

Janazsewska A, Bartosz G (2002). Assay of antioxidant capacity: comparison of four methods as applied to human 
blood plasma. Scand J Lab Invest 62:231-236.

Kopp HP, Kopp CW, Festa A, Krzyzanowska K, Kriwanek S, Minar E, Roka R, Schernthaner G (2003). Impact of weight loss on inflammatory proteins and their association with the insulin. Arterioscler Thromb Vasc Biol 23(6):1042-1047.

Low S, Chew Chin M, Deurenberg-Yap M (2009). Review on epidemic of obesity. Ann Acad Med Singapore 38:57-59

Matthews DR, Hosker JP, Rudenski AS, Naylor AS, Turner RC (1985). Homeostasis model assessment: insulin resistance and beta-cell function from fasting plasma glucose and insulin concentrations in man. Diabetologia 28(7):412-419.

Maury E, Brichard SM (2010). Adipokine dysregulation, adipose tissue inflammation and metabolic syndrome. Molecular and Cellular Endocrinology 314:1-16.

Park J, Chung JJ, Bum Kim J (2007). New evaluations of redox regulating system in adipose tissue of obesity. Diabetes Research and Clinical Practice 77S:S11-S16.

Roberts CK, Sindhu KK (2009). Oxidative stress and metabolic syndrome. Life Sciences 84:705-712.

Sledzinski T, Goyke E, Smolenski RT, Sledzinski Z, Swierczynski J (2009). Decrease in serum protein carbonyl groups concentration and maintained hyperhomocysteinemia in patients undergoing bariatric surgery. Obes Surg 19:321-326.

Stefanović A, Kotur-Stevuljević J, Spasic A, BogavacStanojević N, Bujisić N (2008). The influence of obesity on the oxidative stress status and the concentration of leptin in type 2 diabetes mellitus patients. Diabetes Res Clin Pract 79(1):156-63.

Stephens JW, Khanolkar MP, Bain SC (2009). The biological relevance and measurement of plasma markers of oxidative stress in diabetes and cardiovascular disease. Atherosclerosis 202:321-329.

Tinahones FJ, Murri-Pierri M, Garrido-Sánchez L, GarcíaAlmeida JM, García-Serrao S, García-Arnés J, GarcíaFuentes E (2008). Oxidative stress in severely obese persons is greater in those with insulin resistance. Obesity 17:240-246.

Wang Z, Nakayama T (2010). Inflammation, a link between obesity and cardiovascular disease. Mediators of Inflammation ID535918:1-17.

World Medical Association Declaration of Helsinki (2001). Bulletin of the World Health Organization 79(4):373374.

Wozniak SE, Gee LL, Wachtel MS, Frezza EE (2009). Adipose tissue: The new endocrine organ? A Review Article. Dig Dis Sci 54:1847-1856. 